\title{
DESIGN SPACE EXPLORATION FOR OPTIMISATION OF DAMAGE TOLERANT STRUCTURES
}

\author{
R. Das ${ }^{1}$, R. Jones ${ }^{2}$ \\ ${ }^{1}$ Department of Mechanical Engineering, University of Auckland, Auckland, New Zealand \\ (r.das@auckland.ac.nz) \\ ${ }^{2}$ Department of Mechanical and Aerospace Engineering, Monash University, Clayton, Victo- \\ ria, Australia
}

\begin{abstract}
In parametric design studies, the strength of a structure is often considered as the primary design criteria, and consequently the optimal (best) structural design is often chosen as the one that minimises the maximum stress generated. However, for structures whereby failure is governed by fracture or fatigue, the residual strength, as distinct from stress, needs to be considered as the explicit design objective.

In this study, the design space for the distribution of residual strength for different structural configurations is evaluated to demonstrate the utility of design space exploration for damage tolerance design optimisation. This was illustrated using the problem of the optimum design of a cutout shape under biaxial load. The geometry of the cutout was represented parametrically, and numerous flaws/cracks were assumed to be located along the structural boundaries. The maximum stress intensity factors associated with the flaws along the boundary were evaluated for each cutout geometry. Finite element modelling was used to calculate the stress field, and a semi-analytical method was used for computation of the stress intensity factors. The design surface for residual strength was found to resemble a 'ship hull'. This shape confirms that a design based on residual strength indeed poses a well-behaved optimisation problem, i.e. a well-defined minimum/maximum region exists. The flatness of the design space for residual strength was demonstrated. The optimum values of the stress intensity factor obtained from the design space agreed well with those determined using various optimisation methods in the literature. It is shown that the residual strength optimised shape can be quite different from the corresponding stress optimised solution. This emphasises the need to explicitly consider residual strength as the design objective. It is shown that a design space exploration can provide a systematic way to reduce the weight of a structure by adopting a 'feasible non-optimal' solution that meets the design criteria, rather than aiming for the 'optimal' (best) solution.
\end{abstract}

Keywords: Design space, Shape optimisation, Damage tolerance, Residual strength. 


\section{INTRODUCTION}

The use of light weight structures is of prime consideration in the aerospace industry. This often results in high operational stress levels and increases the likelihood of crack initiation and propagation. This led to the inception of damage tolerance design philosophy in which the presence of cracks and defects in a structure is taken into account. Although first implemented in aerospace industries, the damage tolerance design philosophy has now become an integral part of the standard design practice in many industrial sectors, such as rail, ship building, mining, etc. When optimising structures designed based on damage tolerance, most of the optimisation approaches focus on weight, stress, displacements, frequency, etc. However, in many cases, structures fail by cracking. This generated an increasing emphasis on damage tolerance optimsation, which treats residual strength and fatigue life, as distinct from stress, as the design objectives.

To address this, we previously developed a residual strength optimisation technique based on a heuristic evolutionary algorithm (Fracture ESO in [1]), and applied it for maximising the residual strength of a stringer cutout in a bulkhead, located in pressurised fuselages of transport aircrafts [2]. We subsequently extended another heuristic method (Biological algorithm) for residual strength [3] and fatigue life [4, 5] optimisation of three-dimensional structures.

Design optimisation including damage tolerance parameters is an inherently iterative process. It usually involves analysis of several trial systems in order to identify an acceptable design. In addition to meeting adequate strength, stiffness, and durability requirements, the design must be both cost effective and reliable. It should also take into account the functionality aspects, performance criteria, and manufacturability of the product within available resources. The complex interaction of all these factors often makes it difficult to determine an acceptable design. One challenge often faced by a designer is to automate the evaluation of several potential designs. Structural optimisation techniques can be of immense aid in obtaining a collection of 'preliminary' improved designs that (partially) meet the design specifications.

The aim of the present study is to demonstrate the advantages of design space exploration for durability based design. The effectiveness and utilities of design space exploration in the context of damage tolerance optimisation are demonstrated. In particular, one purpose of this paper is to evaluate the characteristics of the design space for residual strength. The optimum solutions obtained via the design space study are compared with those predicted by different structural optimisation methods.

\section{DESIGN SPACE EXPLORATION}

A design space for structural designs is a collection of structural responses (i.e. the values of objective and constraint functions) for various combinations of structural geometries and/or configurations (i.e. design variables). One way to perform a detailed (iterative) design study is to visualise the partial or the entire design space. The initial step for the design space study is similar to that of optimisation. The 'design problem' is to be cast as an equivalent 
'standard optimisation problem'. This requires that the problem geometry be represented in terms of a set of design variables and the structural responses be chosen as the objective and constraint functions. The design space can then be determined by analysing the structure for each possible combination of the design variables (design point). The behaviour of the design space may be useful in evaluating alternative designs. An overview of the entire design space also helps understand the physical characteristics of the structure and may sometimes reveal a set of designs that may even be preferred over the 'optimum' point itself.

Two broad approaches can be adopted for damage tolerance design optimisation: structural optimisation using (gradient-based or heuristic) optimisation algorithms [1, 4-7] and design space exploration. One advantage of design space visualisation over traditional optimisation methods is that it allows us to focus on innovative designs that may have other useful features, e.g. reduced weight, lower cost, ease of manufacture, that were not explicitly included as part of the standard optimisation objectives. In this paper, we will evaluate the characteristics of the design space of the primary damage tolerance criteria, viz: the residual strength of a structure via a simple example. The design space exploration of fatigue life, another important damage tolerance parameter, will be reported in a future paper.

\section{ILLUSTRATIVE PROBLEM}

\subsection{Problem description}

Design space exploration for damage tolerance parameters is illustrated through the simple problem of 'the optimum design of a cylindrical cutout in a rectangular block under biaxial loading'. This specific problem was selected as it has been used in the previous optimisation studies by the present authors and others in the literature $[3,4,6,7]$, and has a stress based analytical (for the plane stress version) optimal shape [8]. Hence, this will enable us to correlate and compare the 'optimum point(s)' observed in the design space with those obtained using different optimisation methods. The hole geometry was described using a functional form with the major axis length $(b)$ and exponent $(p)$ being considered as the design variables.

The problem geometry, loading and boundary conditions are shown in Figure 1. It is a 3D block, $320 \mathrm{~mm}$ wide, $320 \mathrm{~mm}$ high, with a thickness of $20 \mathrm{~mm}$, and has a cylindrical through-the-thickness cutout at its centre. The diameter of the initial cutout was $20 \mathrm{~mm}$. The material of the block was assumed to be an aluminium alloy (2219-T851) with a Young's modulus of $71 \mathrm{GPa}$ and a Poisson's ratio of 0.3 . Due to symmetry of the structure, a oneeighth model of the block along with the loads and constraints was considered with symmetry conditions imposed on the appropriate planes ( $x z$ and $y z$ planes), as shown in Figure 1. The block was subjected to a uniform tensile stress of $50 \mathrm{MPa}$ on the right and left faces (normal to $x$ axis). The top and the bottom faces (normal to $y$ axis) were subjected to a tensile stress of $100 \mathrm{MPa}$.

The problem serves as a generic example in that the section near a cutout, for many structures, can be modelled as a through-hole in a block under biaxial load. The presence of such 'cutouts' is common in engineering structures used in many industries, such as rail, aerospace, naval, and mining. These 'cutouts' are typically used for lightening the structure or for 
providing passage for equipment and cooling. Hence, it is envisaged that the procedure outlined here to evaluate the characteristics of damage tolerance optimisation via the cutout shape example can be easily extended to durability based shape design of cutouts in similar structures. It should be noted that all the planes $(x y, y z$ and $x z)$ mentioned in the rest of the paper refer to Figure 1.

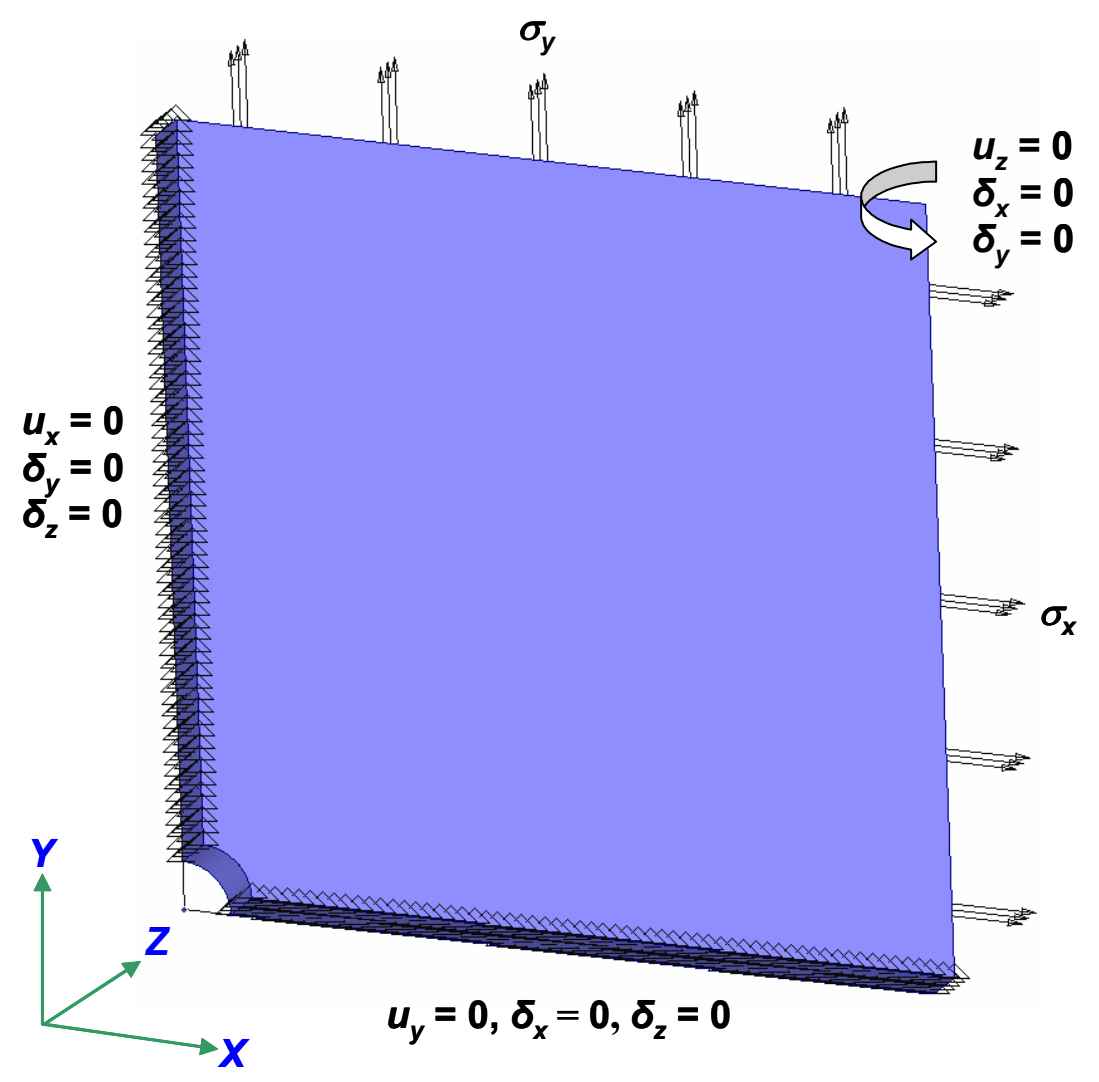

Figure 1. Schematic of the one-eighth model of a cylindrical cutout in a rectangular block under biaxial load.

\subsection{Crack modelling}

A number of surface cracks were modelled on the hole boundary. All the cracks were assumed to be semi-elliptical flaws emanating from the hole surface with their major axes $(c)$ parallel to the axis of the hole ( $z$ axis) and minor axes $(a)$ normal to the hole surface, see Figure 2. An initial crack spacing approximately equal to the smallest crack size was used to achieve an effective modelling of the stress intensity factor (SIF) variation along the structural boundary. Here we modelled 21 three-dimensional semi-elliptical cracks along the surface of the cylindrical hole (for one quarter) resulting in an initial crack spacing of $\sim 0.75 \mathrm{~mm}$.

The parametric position of each crack centre/design point was defined by choosing the top point of the hole (point A in Figure 2) as the origin. The distance of a given point, or crack location, from the origin along the hole boundary curve expressed as a fraction of the total boundary length (curvilinear length from point A to B) was used to define the parametric position of that point, see Figure 2. For the residual strength study the hole surface was assumed to contain semi-elliptical surface flaws along each quarter of the hole with a major axis $c=6$ $\mathrm{mm}$ and a minor axis $a=3 \mathrm{~mm}$. This specific flaw case was chosen because this was the representative case previously studied [3]. 


\subsection{Geometry representation}

The geometric representation of the hole shape in the $x y$ plane is given by:

$$
\frac{x^{p}}{a^{p}}+\frac{y^{p}}{b^{p}}-1=0
$$

where $a, b$, and $p$ are the shape parameters. The shape of the hole is altered by varying these parameters. Any combination of them can be chosen as the design variables for optimisation. As such, this geometric description is ideally suited to the present problem of the optimum design of a cutout in a rectangular block under biaxial loading. Hence, in the present study, Equation 1 was used to generate the design points on the hole surface for a given combination of design variables $(a, b$, and $p)$.

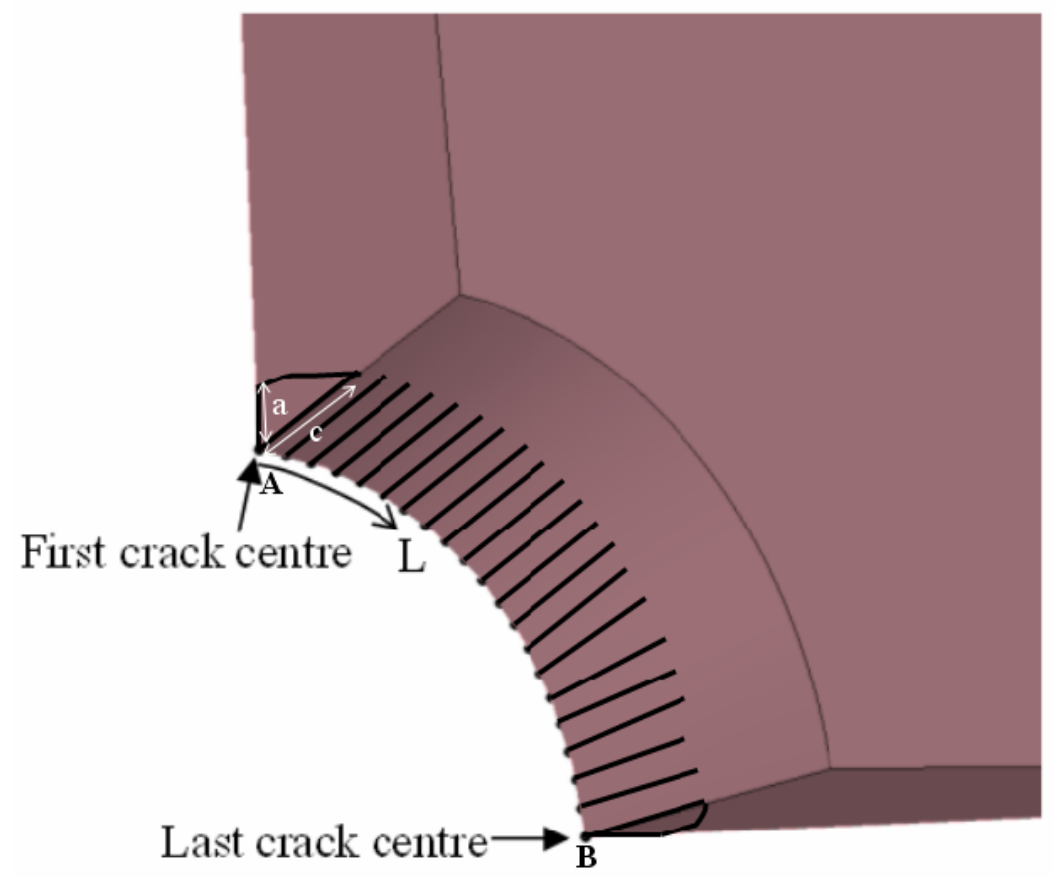

Figure 2. Locations of the 3D semi-elliptical cracks along the hole surface (one-eighth model).

\subsection{Residual Strength Evaluation}

The crack analysis method used here is based on a semi-analytical method proposed by Jones et al. [9]. This semi-analytical method was used to compute the stress intensity factors associated with the cracks on the design (hole) surface. This method can be considered as an amalgam of the Finite Element Alternating Method (FEAM) [10] and a weight function technique. It is implemented in the in-house fracture analysis code FAST (Failure Analysis of STructures). This code uses the displacement field produced by finite element analysis to evaluate the stress field of the uncracked structure. For each crack around the boundary, a set of gauss points are generated on the crack face, and the stresses at these points normal to the crack plane are computed. The stresses are then used to compute the stress intensity factors around the crack front using the FAST code (for details refer to [9]). For 3D cracks, the stress 
intensity factor varies along the crack fronts. The stress intensity factors at the deepest points and the surface points of the 3D flaws are recorded, which are then used to fit a polynomial surface. The surface parameters, i.e. the coefficients of the polynomial, are used to describe the stress intensity factor distribution around the crack fronts. In the present problem, the stress intensity factor was maximum either at the deepest point or at the surface point of a given semi-elliptical crack.

\section{DESIGN SPACE EXPLORATION}

The number of points used to plot a design space is evidently problem dependent. If the objective and constraint functions are 'well-behaved', then a moderate number of regularly spaced points may suffice. The number of design points employed should be suitably chosen so as to obtain an insight into the behaviour of the structure as the geometry changes. However, the computer time is proportional to the number of design points (i.e. geometries) analysed. A trade-off between the computational efficiency and the extent of information required usually dictates this choice. It is instructive to start with a set of regularly spaced design points covering the design space as much as possible. The responses, i.e. the objective and constraint functions, associated with these points are then evaluated. In this way the generic nature of the solution space can be explored. This also helps locate the approximate 'near' optimum zone(s). In the next step, the number of design points can be increased in potential 'localised' optimum regions so as to capture the variation of the objective function with greater clarity.

For residual strength based design, the objective is to minimise the fracture criticality, i.e the maximum stress intensity factor associate with all the cracks present on the design boundary. To investigate the nature of variation of the maximum stress intensity factor associated with different cutout shapes, the design parameters, hole dimension $(b)$ and curvature index $(p)$, were varied to generate a set of design points. The major axis $b$ was varied from 10 to $30 \mathrm{~mm}$ in steps of $0.1 \mathrm{~mm}$, and the exponent $p$ was varied from 2 to 3 in steps of 0.05 . A total of 4221 design points were thus generated. This enabled a reasonably accurate representation of the design space, which was also used for validating the previous optimisation results. The computation was performed using the Victorian Partnership for Advanced Computing (VPAC) Linux cluster super computer. The geometrics (design points) of the structure were generated using one central processor and then distributed into a number of processors, which evaluated the different regions of the design space in parallel. The results from the various processors were subsequently assembled to generate the solution spaces shown in Figures 3-4. The following observations can be made regarding the nature and characteristics of the design space for residual strength for the present problem.

\section{FLATNESS OF THE DESIGN SPACE AROUND THE OPTIMUM POINT}

The design space for the maximum stress intensity factor $\left(K_{\max }\right)$ is shown in Figure 3. The shape of the surface appears like a 'ship hull' with the maximum stress intensity factor 
being higher near both the ends, i.e. for higher and lower values of $b$. The maximum stress intensity factor attained its minimum value of $9.677 \mathrm{MPa} \sqrt{\mathrm{m}}$ at $b=23.1 \mathrm{~mm}$, and $p=2.1$, see Figure 3. It is noteworthy that although a unique optimum shape (i.e. one having the minimum $K_{\max }$ ) could be identified, the values of the maximum stress intensity factor for the surrounding points were also very close to this 'optimum' value. From the design surface (Figure 3 ) and the line contour plot (Figure 4), it can be seen that within a small bounded region around the optimum point, the maximum stress intensity factor was almost constant. In this region it varied from a value of 9.68 to $9.80 \mathrm{MPa} \sqrt{ }$. This again demonstrates that around the optimum point the design space is 'flat'. This means all the shapes in this 'flat' region will have 'nearly' the same maximum stress intensity factor. This is also supported by the earlier findings about the nature of the design space using various optimisation studies $[3,11,12]$. This 'flat' design space can be thought of as a set of local 'optimums' clustered in a small region. Since all of these optimum points have approximately the same value of the objective function $\left(K_{\max }\right)$, it is thus appropriate to conclude from the design spaces that this class of problems has a 'global optimum region' instead of a global optimum point.

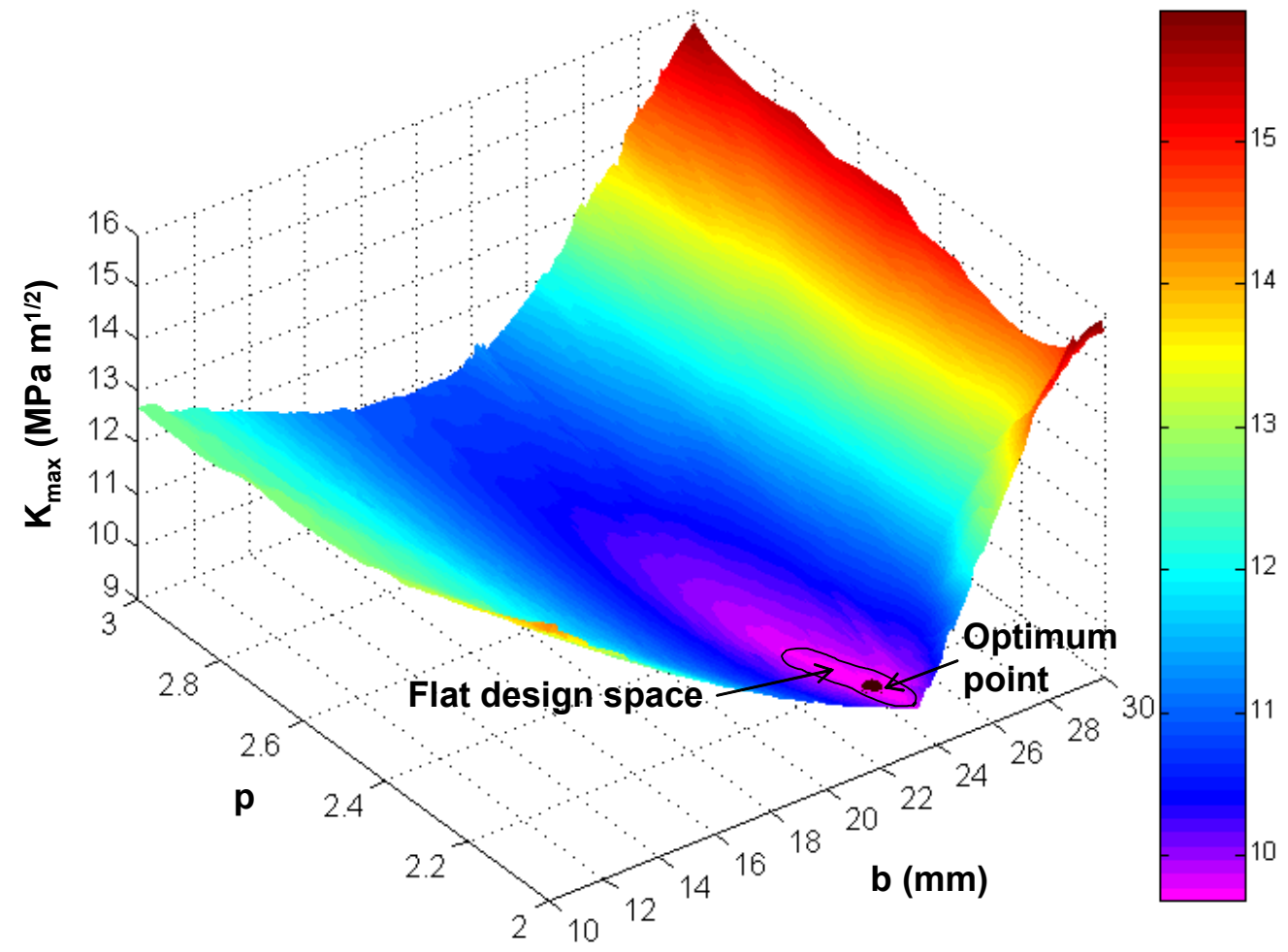

Figure 3. Residual strength study- Design space plot of the maximum stress intensity factor (the objective function) with hole geometric parameters. 


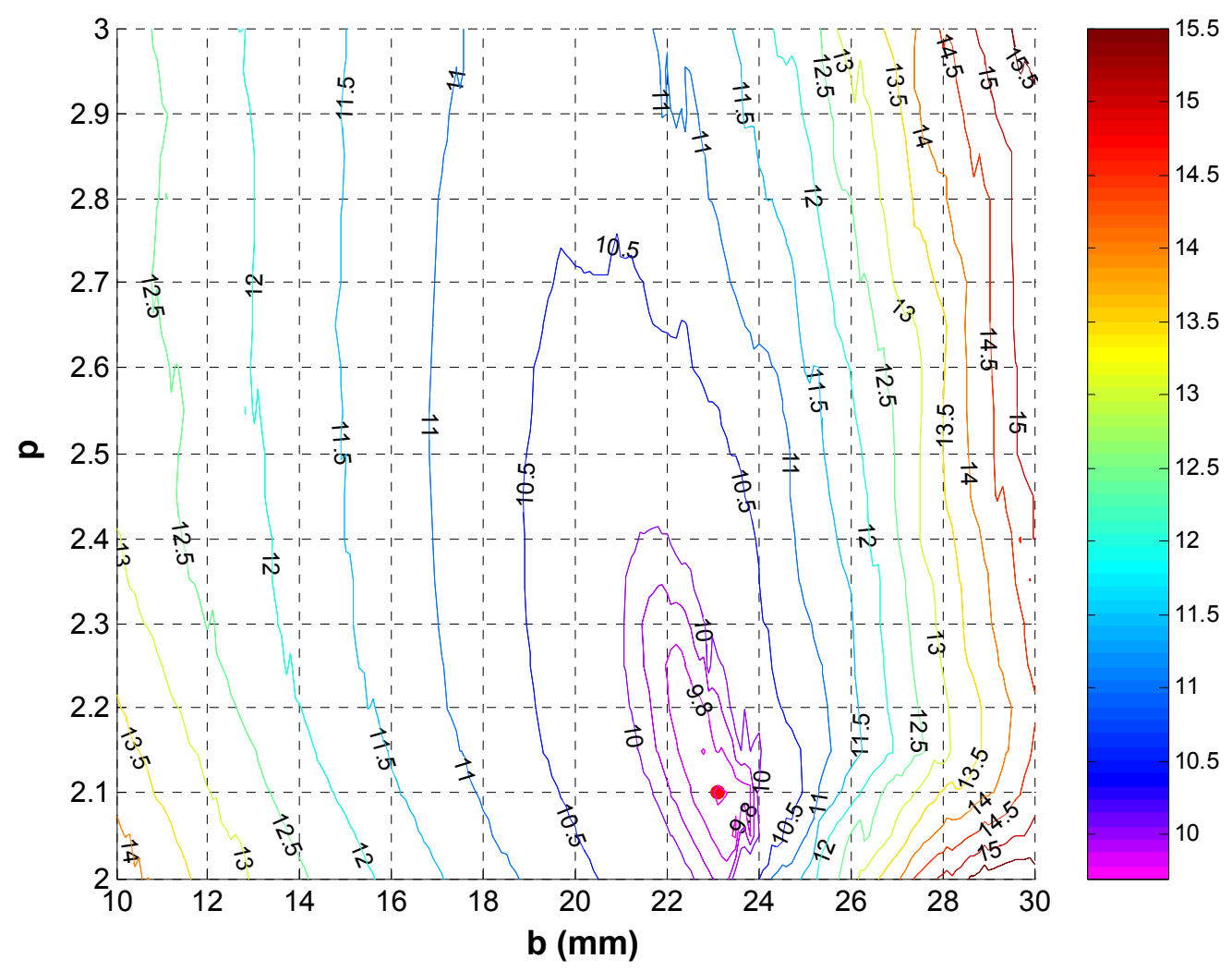

Figure 4. Maximum stress intensity factor $\left(K_{\max }\right)$ distribution with hole dimension $(b)$ and curvature index $(p)$.

\section{RELATIONSHIP OF DESIGN SPACE STUDY WITH STRUCTURAL OPTIMI- SATION}

Design space exploration and optimisation are closely related in that in structural optimisation we move through the design space using an algorithm in order to improve the current design, whereas in a design space study we attempt to obtain an overall view of the variation in the design objective function with structural geometry/shape. Indeed, one of the earlier optimisation algorithms, known as the 'random search method', utilises a similar concept [13]. It is a 'zero-order' method and does not use any gradient information for searching the design space; rather it randomly selects a set of sample points and evaluates the objective function at these points. This forms the basis of further search for an improved design. Thus, instead of a complete analysis of the entire design space, the random search method explores the design space at discrete points and utilises its property to find a 'near' optimum (improved) solution.

For many optimisation problems, the optimum solution may not be unique and often depends on the starting shape, especially if multiple (local) optimum points exist. In such cases, an initial examination of the nature of the design space can help set a starting solution that would (eventually) converge to an improved (local) optimum point. This can lead to a significant improvement in the structural performance in cases where there is a considerable variability among the objective functions associated with the different local optimum shapes. In contrast, for the present problem of the cutout shape design with fracture strength as the 
design objective, an initial design space evaluation can save computational time. The realisation that the design space around the optimum point is 'flat' means that once a design point is in the 'near' optimal zone, any solution in the neighbourhood could be taken as an acceptable design, as the residual strength of the structure will not improve appreciably by further refining the solution to locate the 'precise' optimum point.

Table 1. Comparison of the residual strength optimisation results with the design space study.

\begin{tabular}{|c|c|c|c|}
\hline Methods & $\begin{array}{l}\text { Hole major } \\
\text { axis }(b) \\
(\mathbf{m m})\end{array}$ & $\begin{array}{l}\text { Hole curvature } \\
(p)\end{array}$ & $\begin{array}{l}\text { Objective function } \\
\left(K_{\max }\right) \\
(\mathrm{MPa} \sqrt{\mathrm{m}})\end{array}$ \\
\hline Biological method [3] & 23.166 & - & 9.766 \\
\hline $\begin{array}{l}\text { Nonlinear programming } \\
\text { method [6] }\end{array}$ & 22.947 & 2.129 & 9.677 \\
\hline Design space study & 23.1 & 2.1 & 9.676 \\
\hline
\end{tabular}

Design space analysis can also be used for verifying the reliability and assessing the performance of optimisation algorithms before applying them to design optimisation of a relatively complex structure. In a previous paper, we have implemented a 3D Biological method for residual strength optimisation [3]. The optimisation results found using the Biological method were later validated by optimising the same problem using the 'fundamentally' different gradient-based nonlinear programming optimisation algorithms [6]. From the design space study, the optimum hole size and curvature index were found to be $23.1 \mathrm{~mm}$ and 2.1, respectively. The hole dimensions and curvatures obtained using the different optimisation methods $[3,6]$ are compared with that observed from the design space in Table 1. Given that the resolutions in plotting the design space were $0.1 \mathrm{~mm}$ along $b$ and 0.05 along $p$, it can be inferred that all the optimisation methods performed fairly well in locating the 'near' optimum solution.

\section{ON WEIGHT REDUCTION AND OPTIMUM DESIGN}

For shape optimisation problems, the weight of an optimised structure has not generally been considered as an important design aspect as modifying a shape locally usually has negligible effect on the weight of the overall structure. However, in recent years extensive use of shape optimisation in designing various local features of a given structure has led to consideration of weight as an (additional) design constraint. For example, in the aircraft and rail industries, where weight is a prime concern, saving little weight in each part may mean a significant weight reduction for the entire structure. 
A design space plot can help in lightening a shape by exploring alternative designs. There are cases when the design is deemed to be acceptable, but the structure is thought to be too heavy. This can be illustrated with the present example, whereby the ability to increase the size of the cutout will lead to weight reduction of the resultant structure. Figure 5 presents the volume of the cutout for different shapes. By combining Figures 3 and 5, a design point (different from the optimum) could be chosen that would have a lower weight, yet maintaining an adequate residual strength.

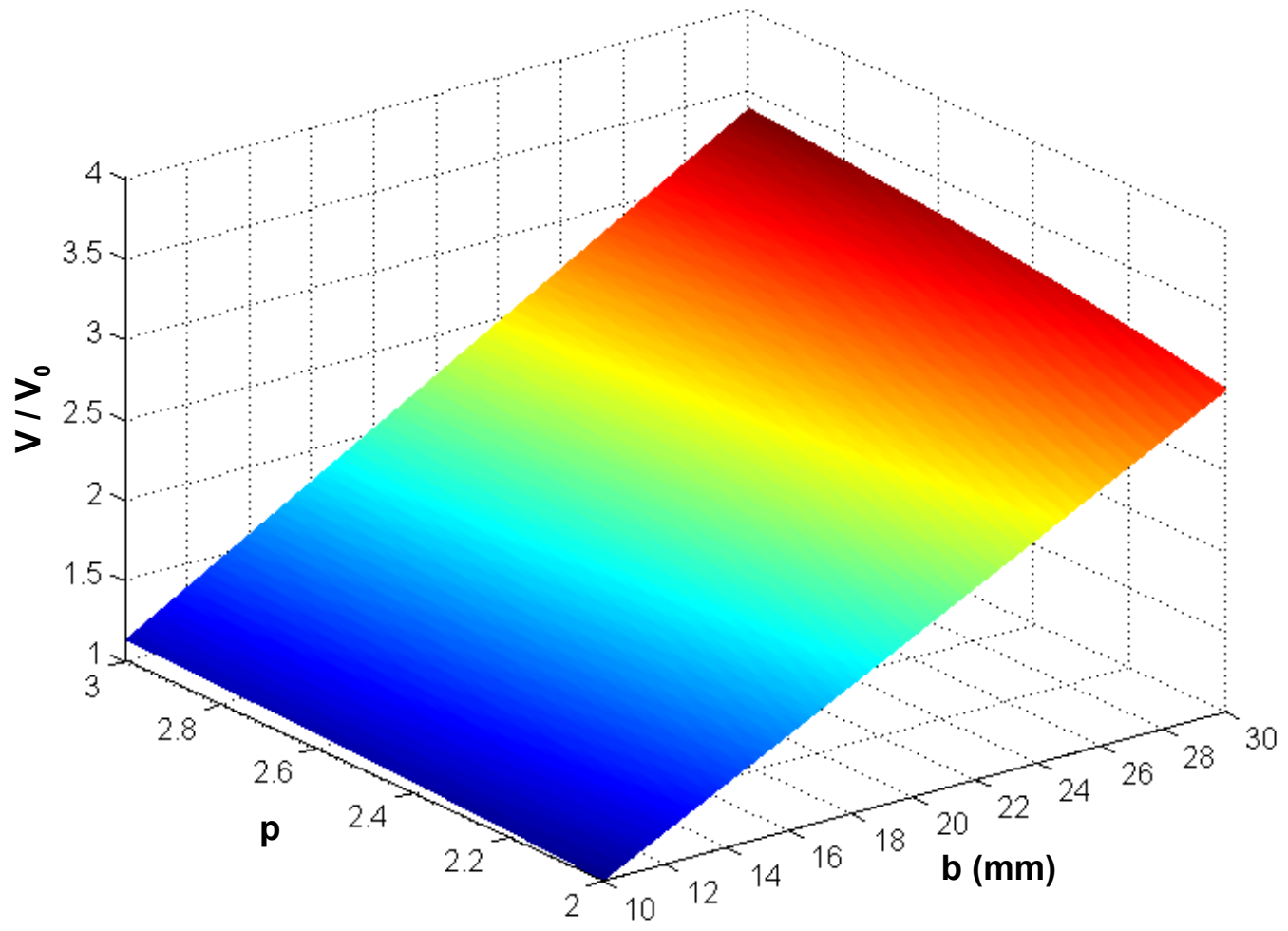

Figure 5: Volume (normalised) of the cutout at various design points.

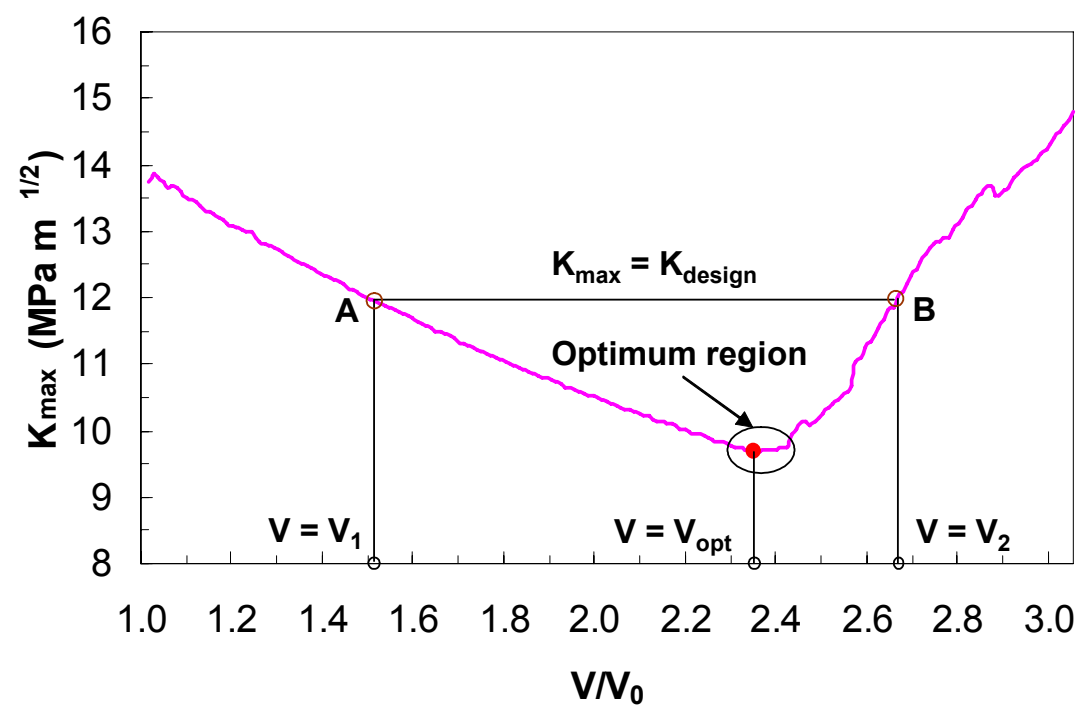

Figure 6: Variation of the maximum stress intensity factor with (normalised) volume for a 'near' elliptical hole with optimum curvature index $\left(p_{\text {opt }}=2.1\right)$.

To illustrate this concept, let us consider a series of cutout shapes, all having the same (optimum) curvature index of $p=2.1$. The variation of the maximum stress intensity factor 
with the volume of the cutout $(V)$ normalised with respect to the volume of a circular hole $\left(V_{0}\right)$ is presented in Figure 6. The maximum stress intensity factor reduced as the hole size increased until the optimum point was reached, after which it increased with further increase in the hole size. At the optimum point we found that: $K_{\max }=9.676 \mathrm{MPa} \sqrt{\mathrm{m}}$, and $V / V_{0}=2.352$. This shows that in addition to reducing the maximum stress intensity factor from 13.82 $\mathrm{MPa} \sqrt{\mathrm{m}}$ (for circular shape) to $9.676 \mathrm{MPa} \sqrt{\mathrm{m}}$, a significant weight reduction ( $\sim 1.35$ times more than that of the circular shape) can be accomplished by implementing the optimum shape.

Now let us suppose that under the present design and operating conditions, the maximum allowable stress intensity factor $\left(K_{\text {design }}\right)$ is $12 \mathrm{MPa} \sqrt{\mathrm{m}}$. In such a scenario, we can use the design space to further lighten the structure as illustrated in Figure 6 . For $K_{\text {design }}=12$ $\mathrm{MPa} \sqrt{\mathrm{m}}$, all the points on the plot below line $\mathrm{AB}$ are 'allowable' or 'feasible' solutions, and any of them may be selected as an acceptable design. If weight reduction is an additional criterion, then instead of choosing the optimum point, adopting the shape corresponding to point B will produce the minimum weight structure satisfying the design criterion $K_{\text {max }} \leq K_{\text {design }}(12$ $\mathrm{MPa} \sqrt{\mathrm{m}}$ ). Indeed, at point $\mathrm{B}, V / V_{0}$ is 2.668 , implying that an additional weight reduction of $\sim 13 \%$ over the optimum shape can be achieved by adopting a 'non-optimal feasible' design.

\section{RELATIVE DOMINANCE OF THE DEEPEST POINT AND SURFACE POINT STRESS INTENSITY FACTORS}

In residual strength based design, the characteristics of the flaws present on the surface being optimised have a crucial role in determining the optimal shape. In the presence of $3 \mathrm{D}$ flaws, the maximum value of the stress intensity factor, i.e. the maximum of the surface point $\left(K_{c}\right)$ and deepest point stress intensity factors $\left(K_{a}\right)$ for all the flaws, drives the optimal solution. In a previous study [3], we found that the relative magnitude of the stress intensity factors at the deepest point and surface point influences the variation of optimal shape with flaw aspect ratio.

This feature can be observed in the design space plot of the maximum stress intensity factors at the deepest and surface points, see Figure 7. For smaller hole size (lower $b$ ), the stress intensity factors at the deepest points $\left(K_{a}\right)$ remain above those at the surface points $\left(K_{c}\right)$. Near the optimum region the domination of the stress intensity factor at the deepest point prevails (i.e. $K_{\max }=K_{a}$ ) for this specific crack case. However, beyond the optimum zone, the two surfaces intersect at a certain region, and the stress intensity factor at the surface point becomes greater. It thus governs the residual strength of the structure thereafter. This can be further elucidated by visualising sections of the design surfaces for $K_{c}$ and $K_{a}$ at planes of constant $p$. Figures 8 and 9 show the variation of $K_{c}$ and $K_{a}$ with $b$ for $p=2.1$ (the optimum curvature), and $p=2$ (elliptical shape), respectively. In Figure 8 the minimum value of the maximum stress intensity factor $\left(K_{\max }\right)$ is equal to $\left(K_{a}\right)_{\min }$ (point B). Here the optimum point is governed by the minimum of either $K_{c}$ or $K_{a}$, whereas in the case of the elliptical hole $(p=2)$ in Figure $9, K_{\max }$ equals neither $\left(K_{c}\right)_{\min }$ nor $\left(K_{a}\right)_{\min }$; rather the minimum $K_{\max }$ occurs at the intersection of the two curves. In this case neither the minimum of $K_{c}$ nor $K_{a}$ drives the minimum point for $K_{\max }$. 


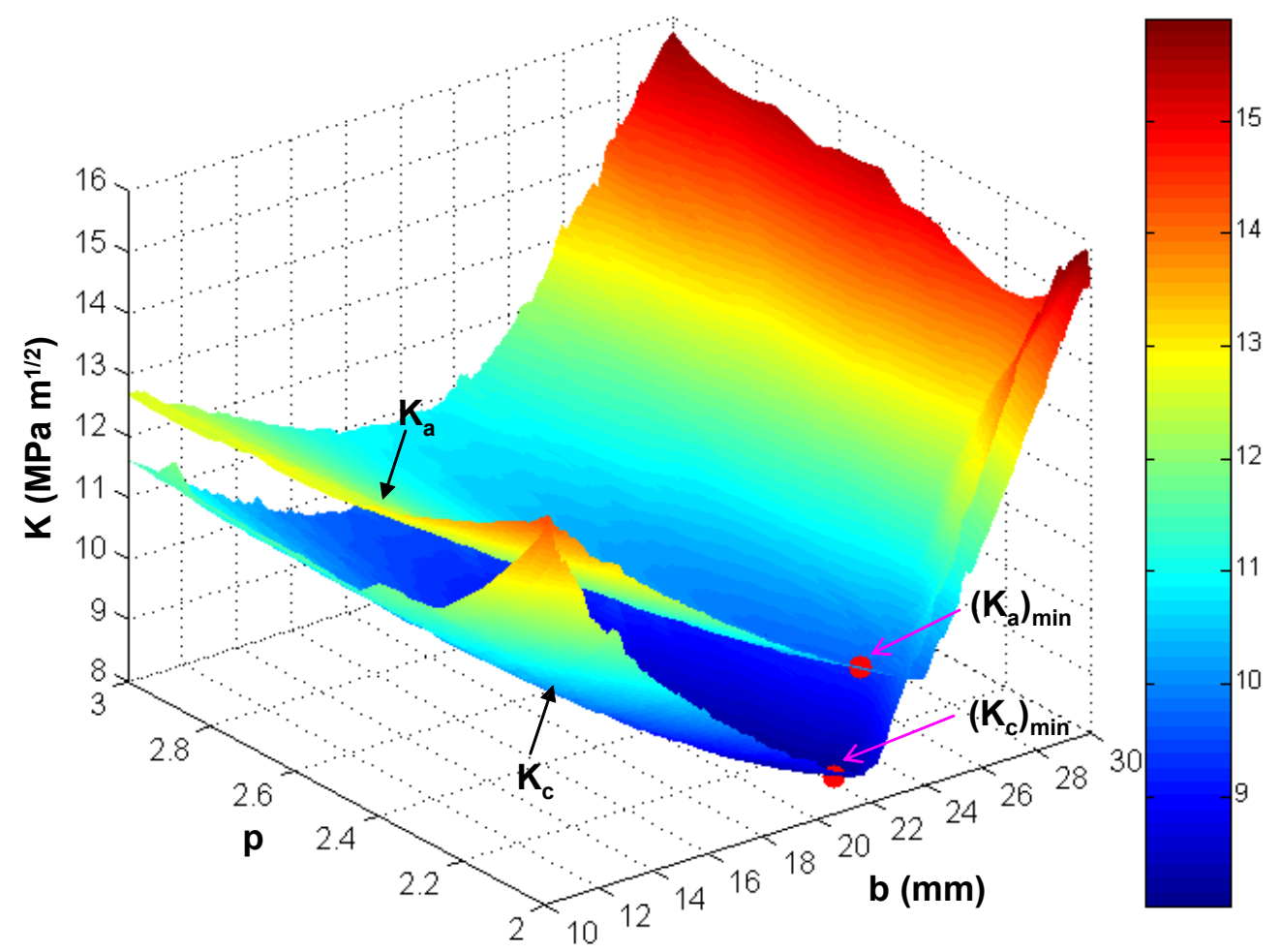

Figure 7: Design space of the maximum stress intensity factors at the surface point $\left(K_{c}\right)$ and deepest point $\left(K_{a}\right)$ with the hole geometric parameters $(b$ and $p)$.

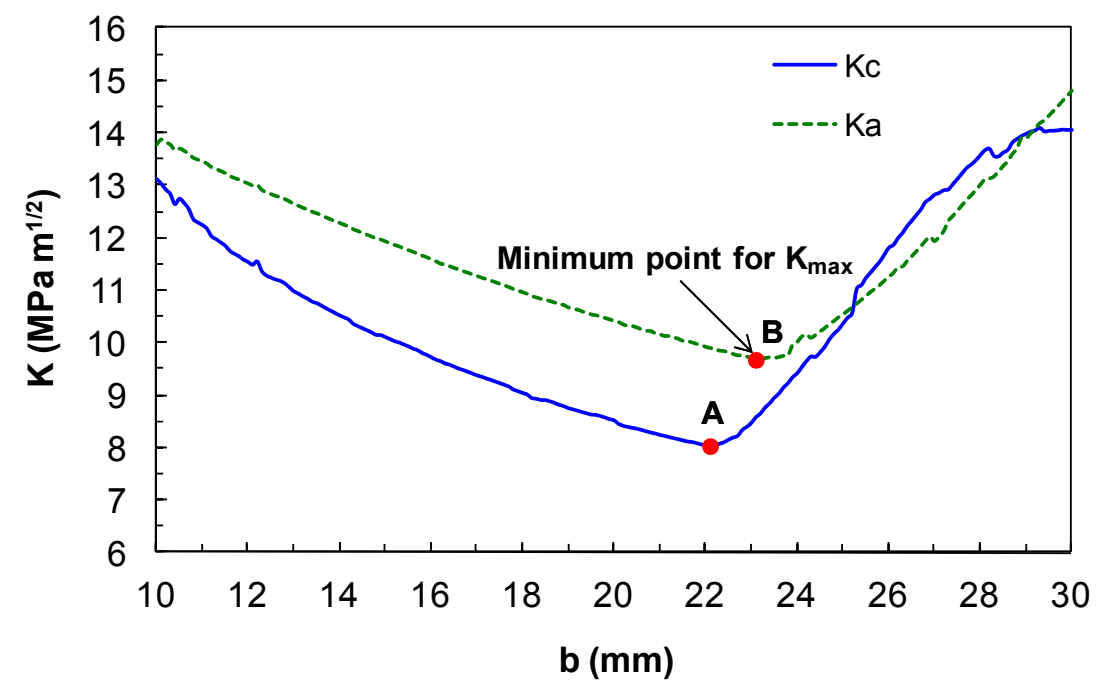

Figure 8: Variation of the maximum stress intensity factors at the surface point $\left(K_{c}\right)$ and deepest point $\left(K_{a}\right)$ with hole size $(b)$ for a 'near' elliptical hole with optimum curvature index ( $p_{\text {opt }}$ $=2.1$ ). 


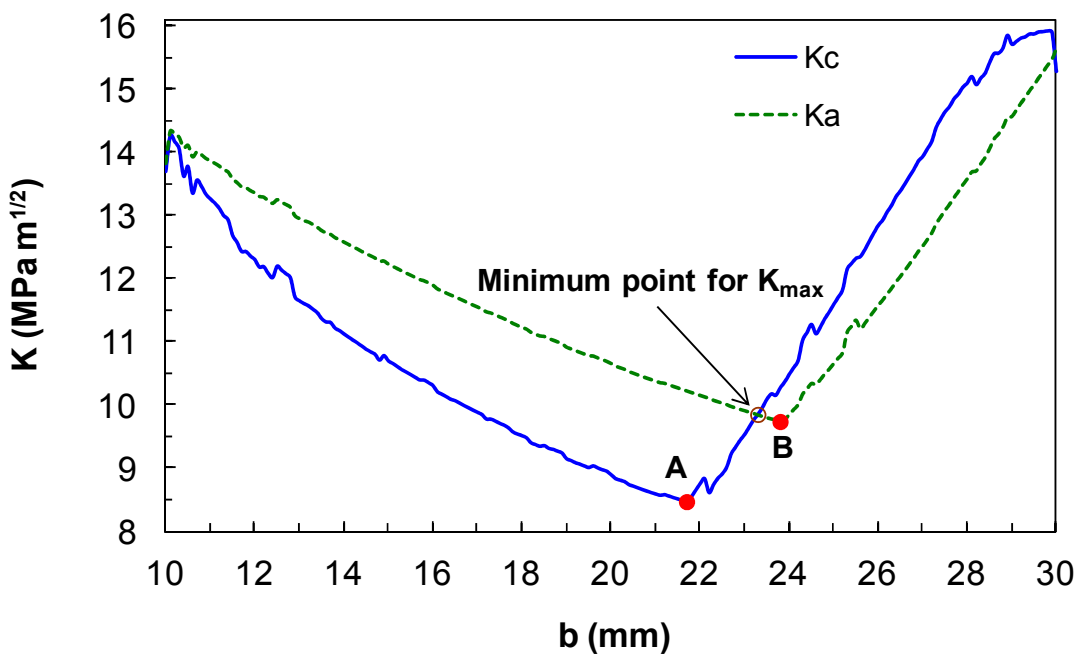

Figure 9: Variation of the maximum stress intensity factors at the surface point $\left(K_{c}\right)$ and deepest point $\left(K_{a}\right)$ with hole size $(b)$ for a perfectly elliptical hole $(p=2)$.

\section{CONCLUSIONS}

In this paper, a design space exploration study has been performed to gain an insight into the variation of the damage tolerance based objective function (residual strength) with structural geometry and to illustrate the utility of design space exploration in the context of design optimisation. The design space study was demonstrated using the problem of the optimum design of a cutout shape under biaxial load. The maximum stress intensity factor associated with the flaws along the structural boundary were evaluated for various hole geometries to construct the design space.

The design surface for residual strength appears to resemble a 'ship hull'. This shape confirms that the design based on residual strength indeed poses a well-behaved optimisation problem, i.e. a well-defined minimum/maximum region exists.

One benefit of a design space study is that it can provide an 'overall view' of the objective function distribution. From earlier studies it was concluded that for this category of problems multiple 'local' optimums can exist. The present study has shown that a set of 'local' optimum solutions can exist in a 'close' neighbourhood, rather than lying apart as found in many other classes of structural optimisation problems. It is therefore contended that this class of damage tolerance optimisation problems has a 'global' optimum region, rather than a single global optimum point. This feature of damage tolerance optimisation has not been previously reported.

The flatness of the design space for residual strength was confirmed, which supported the earlier findings using the various optimisation methods. This means that from an engineering design point of view, the structural responses of various geometries in the 'near' optimal region will not be considerably different. Thus, it may be sufficient to choose one of the shapes in the 'near' optimal region as the final design. This can immensely reduce optimisation effort and computational time, as there will not be any need to precisely locate the (local/global) optimum solution. We can also lighten a structure by removing material appropri- 
ately from a 'near' optimal geometry without significantly degrading its durability related structural performance. Another approach of weight reduction via design space exploration could be by adopting a 'feasible non-optimal' solution that meets the design criteria, rather than aiming for the 'best' solution.

\section{REFERENCES}

[1] Das, R., Jones, R.,Xie, Y.M., "Design of structures for optimal static strength using ESO". Engineering Failure Analysis. 12 (1), 61-80, 2005.

[2] Das, R., Jones, R.,Chandra, S., "Damage tolerance based shape design of a stringer cutout using evolutionary structural optimisation". Engineering Failure Analysis. 14 (1), 118 $137,2007$.

[3] Das, R., Jones, R.,Peng, D., "Optimisation of damage tolerant structures using a 3D Biological algorithm". Engineering Failure Analysis. 13 (3), 362-379, 2006.

[4] Das, R.,Jones, R., "Development of a 3D Biological Method for Fatigue Life based Optimisation and its Application to Structural Shape Design". International Journal of Fatigue. 31 (2), 309-321, 2009.

[5] Das, R.,Jones, R., "Damage tolerance based design optimisation of a Fuel Flow Vent Hole in an aircraft structure". Structural and Multidisciplinary Optimization. 38 (3), 245265, 2009.

[6] Das, R.,Jones, R., "Designing cutouts for optimum residual strength in plane structural elements". International Journal of Fracture. 156 (2), 129-153, 2009.

[7] Das, R.,Jones, R., "Fatigue life enhancement of structures using shape optimisation". Theoretical and Applied Fracture Mechanics. 52 165-179 (DOI: 10.1016/j.tafmec.2009.09.006), 2009.

[8] Kristensen, E.S.,Madsen, N.F., "On the Optimum Shape of Fillets in Plates Subjected to Multiple in plane Loading Cases". International Journal for Numerical Methods in Engineering. 10 1007-1019, 1976.

[9] Jones, R., Peng, D., Pitt, S.,Wallbrink, C., "Weight functions, CTOD, and related solutions for cracks at notches". Engineering Failure Analysis. 11 (1), 79-114, 2004.

[10] Nishioka, T.,Atluri, S.N., "Analytical solutions for embedded elliptical cracks, and finite element alternating method for elliptical surface cracks, subjected to arbitrary loadings". Engineering Fracture Mechanics. 17 (3), 247-268, 1983.

[11] Das, R.,Jones, R., "On the advances of design optimisation of damage tolerant structures". In Proceedings of the International Conference on Advances in Structural Integrity. in Bangalore, India. 2004.

[12] Das, R.,Jones, R., "Designing Structures for Optimum Fracture Strength". In Proceedings of The International Conference on Failure Analysis and Maintenance Technologies. in Brisbane, Australia. 2004.

[13] Vanderplaats, G.N., "Numerical Optimization Techniques for Engineering Design: with Application". New York: McGraw-Hill. 1984. 